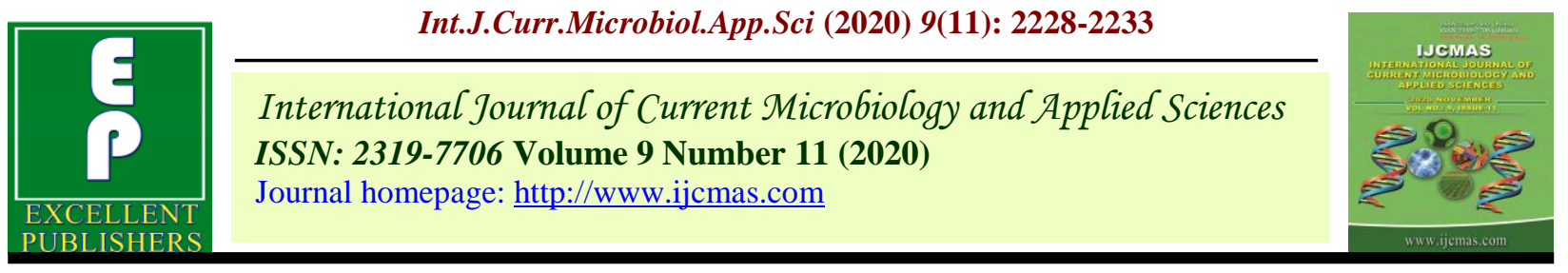

Case Study

https://doi.org/10.20546/ijcmas.2020.911.267

\title{
Management of Intestinal Prolapse Following Blunt Trauma in a Cat- A Rare Case Report
}

\author{
Pipelu Wahengbam*, Naveen Kumar Verma, C. R. Rekha Pathak, \\ Swapna and R. Sasikala
}

Division of Surgery, ICAR-IVRI, Izatnagar, Bareilly, 243122, U.P, India

*Corresponding author

\section{A B S T R A C T}

\begin{tabular}{l} 
Ke y w or d s \\
$\begin{array}{l}\text { Cat, Blunt trauma, } \\
\text { Intestinal Prolapse, } \\
\text { Anastomosis }\end{array}$ \\
\hline Article Info \\
$\begin{array}{l}\text { Accepted: } \\
17 \text { October } 2020 \\
\text { Available Online: } \\
10 \text { November } 2020\end{array}$ \\
\hline
\end{tabular}

\section{Introduction}

Blunt force traumas in small animals are common due to motor vehicle accidents (Taylor et al., 2018) and cause multiple injuries. The other consistently reported cause of accidental blunt trauma in cats is high-rise syndrome, with the most commonly reported abdominal injuries in a recent study noted to be diaphragmatic rupture $(0.02 \%)$, abdominal wall rupture $(0.02 \%)$ and urinary bladder rupture $(0.01 \%)$ (Vnuk et al., 2004). Prolapse is the falling down or slipping of a body part from its usual position or relation which may be due to various reasons like a sudden blow. Rectal prolapse normally occurs in cats due to various cause like neoplasm associated intussusceptions (Demetriou and Welsh, 1999), severe colitis, urogenital diseases, colonic duplication or secondary proctitis due to parasitic infection (Secchi et al., 2012; Carbonell Buj et al., 2020) but intestinal prolapse has not been commonly reported. During intestinal prolapsed, the intestinal part has been slip out from its normal position and lay out of the body through the anal orifice. Exploratory laparotomy is routinely performed in small animal practice and is indicated when organ dysfunction or trauma involving the abdominal cavity requires definitive diagnosis along with surgical treatment and prognosis (Boothe et al., 1992). 
Intestinal anastomosis is commonly performed in veterinary medicine following resection of diseased or devitalized intestinal tissue (Mullen et al., 2020) to restore bowel continuity. In cases of intestinal prolapse, colopexy might facilitate prevention of wandering intestinal loops and further prolapse of the bowel. Colopexy is a surgical procedure used in animals with viable tissue and where manual reduction of the prolapse is difficult (Aronson. 2003). Nevertheless, special care is a must with injured cat, due to increased susceptibility to stress and tendency toward severe hypothermia and shock (Kitchell and Haskins, 1984). So, the present case report describes about the clinical signs, surgical management and outcome of a cat met with a blunt trauma resulting in intestinal prolapse. Moreover, what is novel is that, to our knowledge no such case of intestinal prolapse as a consequence of blunt force trauma has been reported in cats.

\section{Materials and Methods}

\section{History and clinical examination}

A 2 years old domestic shorthair tom cat weighing $3.2 \mathrm{Kg}$ was presented to Referral Veterinary polyclinic, IVRI as an emergency case with the complaint of intestinal tissue protruding from anus. As per the owner the case had a history of unusual trauma, which led to intestinal prolapse, otherwise the pet was normal and active. The owner was unaware of the occurrence resulting in delayed case presentation. The cat was on no sort of treatment at the time of presentation to our polyclinic. The cat was presented in recumbent state with pale the mucus membrane, hypothermic $\left(97^{\circ} \mathrm{F}\right)$ and mild bradycardia (120 beats per minute). The cat appears depressed and other vital parameters were checked. Several prolapsed loops of intestine contaminated with hairs and dirt were visible. Closer examination reveals that the surface of the intestine was pink and moist, while some part of the intestinal mucus membrane looks pale and greyish in colour indicating necrosis of the area due to prolonged exposure to external environment (Fig. 1 and 2).

From the above history and clinical examination the case was diagnosed as prolapsed intestine due to blunt trauma in the abdominal region. The case was considered as an emergency and decided to treat it surgically by laparotomy to reduce further trauma, since per-rectal approach to replace the prolapsed mass was impracticable. Biochemical profile and complete blood count were not obtained keeping in view the emergency of the case.

\section{Pre-operative and surgical treatment}

Appropriate intravenous fluid therapy has been provided to achieve timely correction of dehydration or hypovolaemia. The cat also received thermal support through air warmer to prevent hypothermia. After checking the vital signs of the patient, the case was taken up for laparotomy. Anaesthesia was induced using combination of Inj. Xylazine @ 0.5 $\mathrm{mg} / \mathrm{kg}$ b. wt. and Inj. Ketamine @ $5 \mathrm{mg} / \mathrm{Kg}$ b. wt. mixed in a single syringe and given slowly to effect intravenously. The cat was placed in dorsal recumbency. Before the surgical procedure the prolapsed mass was washed with copious amount of luke warm normal saline solution repeatedly by using gentle scrub to wash off the dirt and hairs from the prolapsed mass. Surgical site preparation was done as per the standard protocol. The hairs on the ventral abdomen were shaved and surgical site was scrubbed using chlorohexidine solution (Savlon) and was painted with povidine iodine before the start of surgery. The cat was positioned and draped in a way that would allow accession of the perineal region if required. 
Midline laparotomy was done by giving skin incision in such a way that it improves access to structures of the caudal abdomen, extending from the umbilicus to pubis. Subcutaneous tissue and fascia separated bluntly and Linea alba was cut to enter the peritoneal cavity. The intestinal mass was slowly pulled back into the peritoneal cavity holding the parts of intestine in the peritoneal cavity while slowly assisting par rectally (Fig. 3). The intestinal mass was explored carefully for perforation and damage. The part of the intestine showing necrosis and perforation with loss of tissue viability was resected (Fig. 4). After resection, viable intestinal parts were anastomosed with simple interrupted suture pattern using absorbable suture material polyglycolic acid (PGA) No.0 in approximating pattern, making sure that the anastomosis include the submucosal layer (Fig. 5) (Hedlund and Fossum, 2007). Any leakage was checked by milking through the anastomosed section. Further colopexy of the intestinal part was done by fixing the colon to the abdominal wall using sutures to create adhesion thus, prevent recurrence of the condition and displacement of the large intestine (Fig. 6).

The abdominal cavity was lavage using large volumes of warm normal saline, which aids in removal of contaminants and patient warming. The abdominal cavity was then closed in three layers. The abdominal wall was closed using the external leaf of the rectus abdominis muscle sheath in a simple interrupted suture pattern using PGA No. 0. Dead space obliterated and the second layer, subcutaneous closure was accomplished in a simple continuous pattern using absorbable suture material. Skin was closed by cross mattress pattern using Polyamide black (Trulon) size 0. Post operatively, Analgesics/anti-inflammatory and antibiotic therapy i.e., Inj. Meloxicam @ 0.2 mg/kg, IM for 5 days; Inj. Ceftriaxone @ $10 \mathrm{mg} / \mathrm{kg}$ and
Inj. Metronidazole @ 30 mg/kg, IV for 7 days was given. Owner was advised to avoid oral feeding for the cat for next 3-5 days and maintain on intravenous fluids. Soft and liquid diet was advised in the later part. Surgical skin sutures were removed after a period of 12 days i.e. after complete healing of the incision site. The patient had an uneventful recovery.

\section{Results and Discussion}

Blunt force trauma mainly due to automobile accidents are common in small animals (Taylor et al., 2018) and cause multiple injuries at different parts of the body. The cat in this report showed prolapsed intestinal loops from the anal orifice which was suspected to be caused by blunt force trauma on the abdomen of the cat, but with limited reports it is unclear to say blunt force trauma on abdomen causes intestinal prolapse. Traumatic injury, rarely affects the gastrointestinal tract owing to its central location and extreme mobility within the abdominal cavity (Cripps and Copper, 1997). Taylor et al., (2018) reported intestinal perforation as a consequence of blunt force trauma in a cat which was operated initially for inguinal hernia with no evidence of intestinal pathology at the time of initial abdominal exploration and later developed septic peritonitis. Exposure of the bowel loops to the external environment might cause contamination and intestinal hypoperfusion that could have aggravate the condition of the intestinal loops causing bowel compromise, necrosis and devitalisation. Considering the emergency of the case, under general anaesthesia with Xylazine- Ketamine combination at low doses laparotomy was performed since the prolapsed intestinal loops cannot be retracted per-rectally. Xylazine and Ketamine combination are used in feline practice for routine surgical procedures and used preferably intravenously for shorter 
duration of anaesthesia and better discrimination of dosage requirement (Dyson and Allen, 1991). The devitalised perforated intestinal part which no longer shows the

signs of healing requires surgical resection and removal which was performed in the present case.

Fig.1 Cat presented with prolapsed bowel from the anal orifice

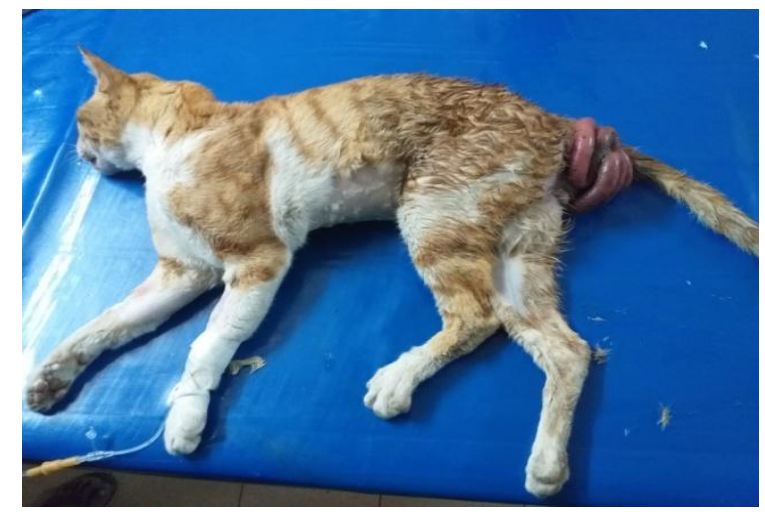

Fig.2 Prolapsed loops of intestinal mass contaminated with dirt and hairs

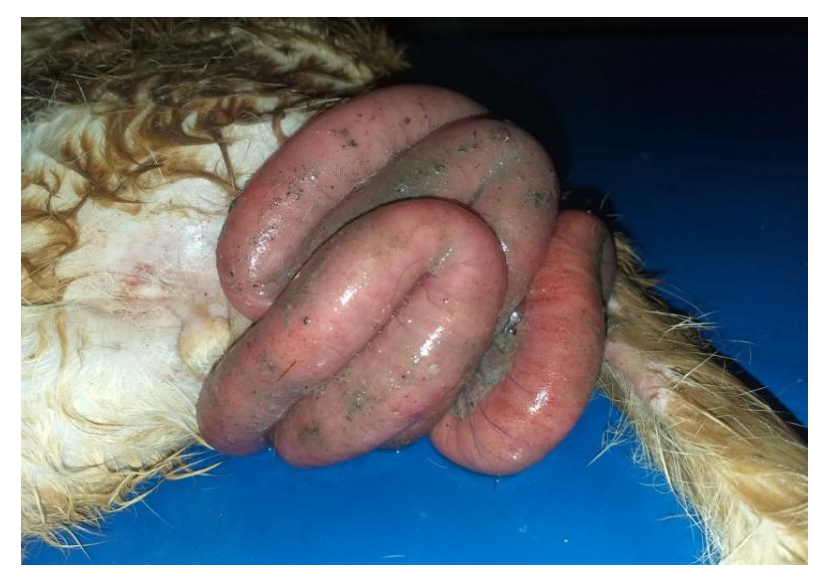

Fig.3 Whole prolapse bowel along with necrosed intestinal tissue section retracted back to the abdominal cavity

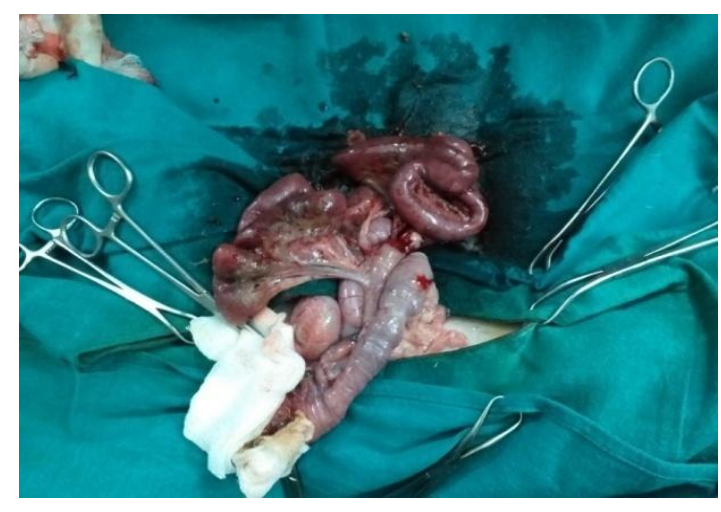


Fig.4 Surgical excision of the necrosed intestinal section

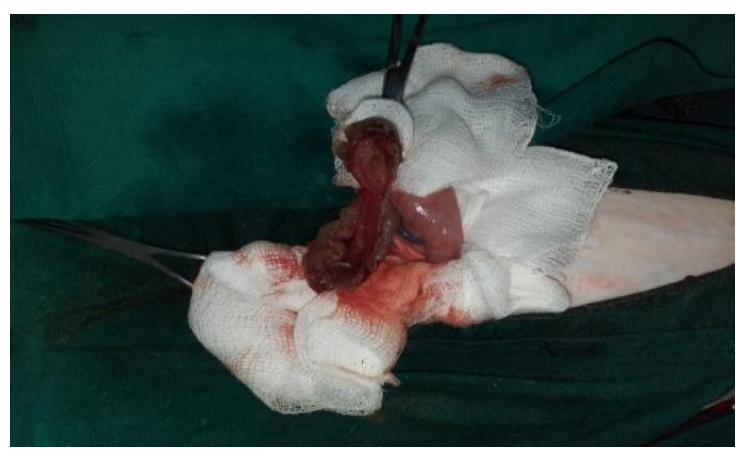

Fig.5 The healthy intestinal tissue anastomosed with simple interrupted suture pattern

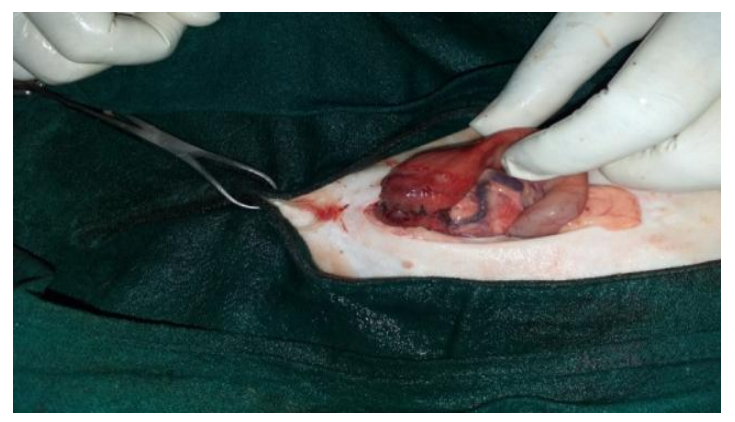

Fig.6 Colopexy of the intestinal tissue to the abdominal wall

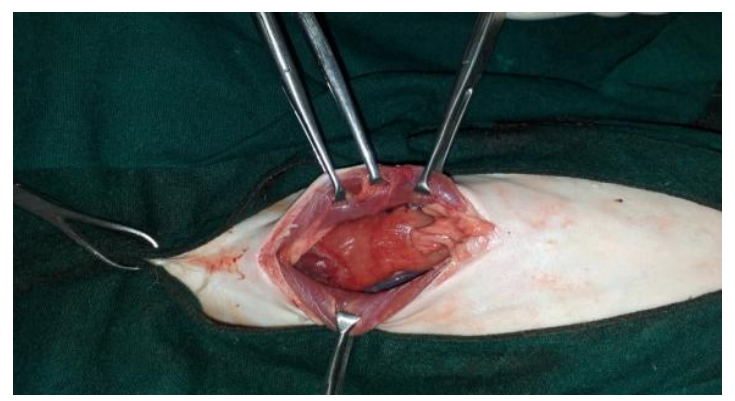

The healthy sections of intestinal loops were anastomosed and healing might have occurred by normal healing process characterized by an initial lag or inflammatory phase, an intermediate proliferative phase, and a final maturation phase (Mullen et al., 2020). Colopexy permits a permanent adherence between the serosal surface of the organ and the abdominal wall, in order to prevent caudal movement of the colon and rectum. Incisional method of colopexy, with laparoscopic access has been successfully used to treat recurrent rectal prolapse (Secchi et al., 2012). In the present case, colopexy has been performed to prevent further prolapse and maintain the position of the bowel in its normal location post surgery. A proper post operative care including antibiotic therapy, analgesia, maintenance of normal body temperature, adequate provision of nutrition, monitoring of the abdominal wound and prevention of patient interference assisted in uneventful recovery of the patient.

In conclusions the present report describes about the blunt trauma leading to intestinal prolapse with the approach for excision of a necrotic tissue from prolapsed intestinal loop 
and anastomosis of the healthy part in a cat and further fixing it with colopexy. The described technique might provide a successful outcome and may increase the success rate of such cases. Such surgical resection appears as the only treatment of a necrotic tissue mass from prolapsed intestine via a laparotomy approach with a promising outcome. Colopexy prevent recurrence of the condition and with sound nursing measures no postoperative complications was observed in our case.

\section{References}

Aronson L. 2003. Rectum and anus. In: D Slatter, ed. Textbook of small animal surgery, 3rd edn. Philadelphia, PA: Saunders. 682-707.

Boothe, H.W., Slater, M.R., Hobson, H.P., Fossum, T.W. And Jung, C., 1992. Exploratory celiotomy in 200 nontraumatized dogs and cats. Vet Surg. 21(6): 452-457.

Carbonell Buj, E., Billet, J.P., Vanel, M. and Caron, A., 2020. Rectal duplication in an adult cat: a novel transanal surgical approach. JFMS Open Rep. 6(1): 2055116920916956.

Cripps NP and Cooper GJ. 1997. Intestinal injury mechanisms after blunt abdominal impact. Ann R Coll Surg Engl. 79: 115-120.

Demetriou, J.L. and Welsh, E.M., 1999. Rectal prolapse of an ileocaecal neoplasm associated with intussusception in a cat. J Feline Med Surg. 1(4): 253-256.
Dyson, D.H. and Allen, D.G., 1991. Injectable anesthetic agents for cats. Can Vet J. 32(5): 314.

Hedlund, CS, Fossum, TW. 2007. Surgery of the digestive system. In: Fossum, TW, ed. Small animal surgery, 3rd edn. St Louis, MO: Mosby. Pp. 339-530.

Kitchell, B.E. and Haskins, S.C., 1984. Feline trauma and critical care medicine. Vet Clin North Am Small Anim Pract. 14(6): 1331-1344.

Mullen, K.M., Regier, P.J., Ellison, G.W. and Londoño, L., 2020. A Review of Normal Intestinal Healing, Intestinal Anastomosis, and the Pathophysiology and Treatment of Intestinal Dehiscence in Foreign Body Obstructions in Dogs. Top Companion Anim Med. 100457.

Secchi, P., Filho, H.C.K., Scussel Feranti, J.P., de Oliveira, M.T., Gottlieb, J., Guedes, R.L., Colomé, L.M. and Brun, M.V., 2012. Laparoscopic-assisted incisional colopexy by two portals access in a domestic cat with recurrent rectal prolapse. J Feline Med Surg. 14(2): 169-170.

Taylor, A., Cooper, E. and Ham, K., 2018. Delayed intestinal perforation secondary to blunt force abdominal trauma in a cat. JFMS Open Rep. 4(1): 2055116918763410.

Vnuk, D., Pirkić, B., Matičić, D., Radišić, B., Stejskal, M., Babić, T., Kreszinger, M. and Lemo, N., 2004. Feline high-rise syndrome: 119 cases (1998-2001). J Feline Med Surg. 6(5): 305-312.

\section{How to cite this article:}

Pipelu Wahengbam, Naveen Kumar Verma, C. R. Rekha Pathak, Swapna and Sasikala, R. 2020. Management of Intestinal Prolapse Following Blunt Trauma in a Cat- A Rare Case Report. Int.J.Curr.Microbiol.App.Sci. 9(11): 2228-2233. doi: https://doi.org/10.20546/ijcmas.2020.911.267 